\title{
Éditorial: \\ La formation en gériatrie influence-t-elle la décision des médecins de s'impliquer dans les soins aux personnes âgées?
}

Trois faits sont déja bien connus: le vieillissement de la population canadienne et ses impacts organisationnels, financiers et éthiques sur les services de santé; la pénurie de médecins intéressés à la pratique gériatrique: le manque de formation des médecins en médecine gériatrique.

En effet, il existe au Québec un problème important dans le recrutement de médecins désireux de pratiquer la gériatrie dans les institutions pour personnes âgées ou dans les programmes communautaires de maintien à domicile. La gériatrie, comme spécialité médicale, ne semble pas encore très attrayante pour les futurs médecins (Duthie et al., 1987). L'enseignement de la gériatrie a été longtemps, comme le souligne Somers, l'un des quatre orphelins de l'éducation médicale avec la médecine préventive, l'économie de la santé et l'éthique médicale (Somers, 1987). Ceci se perçoit dans les difficultés qu'éprouvent les médecins à dépister et à diagnostiquer parmi leur clientèle âgée certains problèmes (tels la perte d'autonomie, la démence, l'incontinence urinaire ou la dépression) (Siu et al., 1988; Beaulieu et Leclère, 1991), à investiguer (Radecki et al., 1988) ou à traiter certaines affections (Larson et al., 1991; Greenfield et al., 1987).

Dans un article de ce même numéro, Judith Globerman nous amène à réfléchir aux facteurs qui influencent la décision des médecins de s'impliquer dans les soins aux personnes âgées. Elle mentionne particulièrement la personnalité et les styles de pratique des médecins, leurs contacts personnels avec les personnes âgées ainsi que leurs expériences éducatives.

Plusieurs questions se posent. Les connaissances et les attitudes face aux personnes âgées modifient-elles la décision des futurs médecins d'exercer auprès des personnes âgées? Est-il possible par la formation en gériatrie de modifier les connaissances et les attitudes chez les étudiants en médecine? Quels sont les déterminants du succès des programmes de formation?

La recension des écrits effectuée tour à tour par Coccaro, Adelman et Beissner est particulièrement éclairante (Coccaro et Miles, 1984; Adelman et Albert, 1987; Beissner, 1990). Les résultats sont parfois contradictoires ou démontrent le faible impact de l'enseignement sur le changement des attitudes des étudiants et de leurs désirs de développer une pratique gériatrique. Ces résultats sont fortement influencés par la méthodologie et les outils de mesure utilisés. Les instruments les plus employés pour évaluer les connaissances et les attitudes envers les personnes âgées présentent plusieurs lacunes: les concepts mesurés varient d'un instrument à l'autre et 
rendent la comparaison difficile; le contenu mesuré est parfois périmé; les instruments sont peu sensibles aux changements; des variables importantes agissant sur le désir des médecins de soigner des patients âgés n'y sont pas intégrées; peu d'études bénéficient de groupes de contrôle; les changements d'attitudes sont mesurés à court terme et rarement à long terme. Ainsi peu d'études vérifient si les désirs de pratique des étudiants auprès des personnes âgées se concrétisent par la suite.

Cependant, de grandes tendances se dégagent de ces études. Il est possible, par un programme d'enseignement bien planifié, d'augmenter les connaissances des étudiants en médecine sur le vieillissement. De plus, il semble exister un lien positif entre l'acquisition de ces connaissances et le développement d'attitudes favorables envers les personnes âgées (Linn et Zeppa, 1987).

Lors de leurs premières années de formation, les attitudes des étudiants en médecine face aux personnes âgées sont en général relativement positives. Par la suite, les étudiants sont soumis à une série de stress reliés tant au déroulement du curriculum de leurs études, qu'aux difficultés organisationnelles de la médecine, qu'aux aspects affectifs soulevés par la confrontation à la maladie chronique et à la souffrance. L'exposition à une formation en gériatrie où une approche globale et humaniste du patient est valorisée et pourrait freiner ce phénomène, du moins à court terme.

Certains facteurs influencent plus particulièrement les attitudes des étudiants envers les personnes âgées et leur désir de pratique gériatrique. Les expériences antérieures avec des personnes âgées, surtout lorsqu'elles ont été positives, constituent un élément important (Beissner, 1990; Green et al., 1983; Adelman et Albert, 1987; Linn et Zeppa, 1987). Il peut s'agir d'expériences personnelles - comme un contact avec un grand-parent, un travail d'été dans un centre d'accueil - ou de rencontres de personnes âgées lors de stages cliniques. Les éducateurs s'accordent sur l'importance de privilégier des contacts favorisant, non pas le nombre mais la qualité, supposant un bon encadrement des étudiants tant au plan cognitif qu'affectif.

Dès les premières années de formation de médecine, les étudiants doivent être invités à rencontrer des personnes âgées; ces contacts peuvent même encore être plus précoces à travers des programmes inter-générations des études primaires, secondaires et collégiales. Les étudiants en médecine doivent également échanger avec des personnes âgées de divers niveaux d'autonomie fonctionnelle afin de saisir l'hérogénéité de la population âgée et de briser le stéréotype de la personne âgée fortement handicappée véhiculé par les milieux hospitaliers. Une diversité de milieux de stage s'impose: domicile de la personne âgée, centre communautaire et centre de jour, programmes de maintien à domicile, centre d'accueil, hôpitaux. Cette exposition doit s'étaler tout au long de la formation.

La personnalité des étudiants est aussi une variable importante. Les étudiants plus ouverts à une approche globale et humaniste de la médecine seraient plus enclins à pratiquer auprès des personnes âgées. Seul le processus de sélection des étudiants en médecine peut amener à privilégier ce profil 
de personnalité. La sélection doit donc se baser non seulement sur le niveau de réussite académique mais également sur l'exploration des personnalités, des valeurs et des attitudes des étudiants. L'Université de Montréal s'est engagée dans cette voie il y a quelques années.

La valorisation personnelle obtenue en soignant les personnes âgées semble être également déterminante (Green et al., 1983). Cette valorisation se développe au contact de médecins dont le modèle de rôle est stimulant tant au plan intellectuel qu'au plan humain. Actuellement la pénurie de gériatres et de médecins de famille intéressés à la gériatrie oblige ceux-ci à assurer en priorité les soins aux personnes âgées et à délaisser leur mission académique, en particulier la recherche et les publications (Siu et al., 1989; Vivell et al., 1987). Ceci ralentit l'avancement de la discipline et ne favorise pas pour le moment le développement de modèle de rôle aussi prestigieux que dans d'autres spécialités établies depuis de nombreuses années.

Également les milieux où oeuvrent ces médecins doivent supporter et encourager leurs médecins enseignants. Les milieux d'enseignement les plus propices à la pratique de la gériatrie se caractérisent souvent par une ouverture à l'interdisciplinarité, non seulement des disciplines médicales entre elles mais avec les autres disciplines gérontologiques, une ouverture à l'humanisme et à une approche communautaire. Le modèle de l'Université de Nottingham est particulièrement inspirant à cet effet (Wattis et al., 1986).

Parmi les autres facteurs qui influencent les médecins dans leur choix de pratique et qui dépendent moins de la formation, soulignons la complexité de la pratique gériatrique au plan clinique et organisationnel; la rémunération qui ne témoigne pas toujours des efforts consentis par le médecin auprès de la clientèle gériatrique (Barker et Williamson, 1986).

Plusieurs études ont démontré, tant aux États-Unis qu'au Canada, que les programmes de formation en gériatrie sont loin d'être implantés partout et que plusieurs ne sont pas encore obligatoires (Reuben et al., 1990; Vivell et al., 1987; Association médicale canadienne, 1987). Cependant comme le souligne Besdine, la gériatrie évolue rapidement depuis quelques années et se dirige vers une maturité en tant que discipline (Besdine, 1989). Au Québec, au cours des dix dernières années, des efforts considérables ont été faits pour développer dans les quatre facultés de médecine un programme d'enseignement de la gériatrie du niveau pré-gradué au niveau post-gradué des études médicales. Une diversité de moyens pédagogiques ont été implantés.

Il faut sans nul doute témoigner d'une certaine patience afin de voir mûrir les fruits de cet enseignement. Cependant, la pression de la démographie conjuguée à la démographie médicale constituera peut être des moteurs plus puissants que l'enseignement dans le choix des médecins pour la pratique gériatrique. Mais la formation des médecins demeurera toujours garante de la qualité de la pratique qu'ils exerceront auprès de leurs patients âgés. Et cette formation ne pourra se réaliser sans la constitution d'un noyau optimal d'enseignants supportés par des milieux universitaires valorisant la gériatrie. 


\section{Références}

Adelman, R.D., \& Albert, R.C. (1987). Medical Student Attitudes Toward the Elderly: A Critical Review of the Literature. Gerontology and Geriatrics Education, 7, 141-155.

Association médicale canadienne. (1987). Les soins de santé pour gens âgés. Deuxième partie. Canadian Medical Association Journal, 137, 544c-544d.

Barker, W., \& Williamson, J. (1986). Survey of Recently Appointed Consultants in Geriatric Medecine. British Medical Journal, 293, 896-898.

Beaulieu, M.-D., \& Leclère, H. (1991). Nature des difficultés de la pratique médicale: les omnipraticiens s'expriment. Bulletin. Corporation professionnelle des médecins du Québec, 31, 17-22.

Beissner, K. (1990). Health Professional Students Intentions' Toward Geriatric Specialization: A Review of Contributing Factors. Gerontology and Geriatrics Education, 10, 71-89.

Besdine, R.W. (1989). The Maturing of Geriatrics. New England Journal of Medecine, 320, 181-182.

Coccaro, E.F., \& Miles, A.M. (1984). The Attitudinal Impact of Training in Gerontology/Geriatrics in Medical School: A Review of the Literature and Perspective. Journal of the American Geriatrics Society, 32, 762-768.

Duthie, E.H., Donnelly, M.B., \& Kirsling, R.A. (1987). Fourth-Year Students' Preference for Geriatrics as a Career. Journal of Medical Education, 62, 511-514.

Green, S.K., Keith, K.J., \& Pawlson, L.G. (1983). Medical Students' Attitudes Toward the Elderly. Journal of the American Geriatrics Society, 31, 305-309.

Greenfield, S., Blanco, D.M., \& Elashoff, R.M. (1987). Patterns of Care Related to Age of Breast Cancer Patients. Journal of the American Medical Association, 257, 2766-2770.

Larson, D.B., Lyons, J.S., \& Hohmann, A.A. (1991). Psychotropics Prescribed to the US Elderly in the Early and Mid 1980s: Prescribing Patterns of Primary Care Practitioners, Psychiatrists, and Other Physicians. International Journal of Geriatric Psychiatry, 6, 63-70.

Linn, B.S., \& Zeppa, R. (1987). Predicting Third Year Medical Students' Attitudes Toward the Elderly and Treating the Old. Gerontology and Geriatrics Education, 7, 167-175.

Radecki, S.E., Kane, R.L., \& Solomon, D.H. (1988). Are Physicians Sensitive to the Special Problems of Older Patients? Journal of the American Geriatrics Socie$t y, 36,719-725$.

Reuben, D.B., Fink, A., \& Vivell, S. (1990). Geriatrics in Residency Programs. Academic Medecine, 65, 382-387.

Siu, A.L., Ke, G.Y., \& Beck, J.B. (1989). Geriatric Medicine in the United States. The Current Activities of Former Trainees. Journal of the American Geriatrics Society, 37, 272-276.

Siu, A.L., Leake, B., \& Brook, R.H. (1988). The Quality of Care Received by Older Patients In 15 University-Based Ambulatory Practices. Journal of Medical Education, 63, 155-161.

Somers, A.R. (1987). Four "Orphan" Areas in Current Medical Education: What Hope for Adoption? Family Medicine, 19, 137-140.

Vivell, S., Solomon, D.H., \& Beck, J.C. (1987). Medical Education Responds to the 20th Century's Success Story. Journal of the American Geriatrics Society, 35, 1107-1115. 
Wattis, J.P., Smith, C.W., \& Binns, V. (1986). Medical Students' Attitudes to Old People and Career Preference: a Comparison of Two Universities. Medical Education, 20, 498-501.

Paule Lebel 